TRANSACTIONS OF THE

AMERICAN MATHEMATICAL SOCIETY

Volume 363, Number 12, December 2011, Pages 6219-6244

S 0002-9947(2011)05490-5

Article electronically published on July 22, 2011

\title{
ON PERMUTATIONS OF HARDY-LITTLEWOOD-PÓLYA SEQUENCES
}

\author{
CHRISTOPH AISTLEITNER, ISTVÁN BERKES, AND ROBERT F. TICHY \\ Dedicated to the memory of Walter Philipp
}

\begin{abstract}
Let $\mathcal{H}=\left(q_{1}, \ldots, q_{r}\right)$ be a finite set of coprime integers and let $n_{1}, n_{2}, \ldots$ denote the multiplicative semigroup generated by $\mathcal{H}$ and arranged in increasing order. The distribution of such sequences has been studied intensively in number theory, and they have remarkable probabilistic and ergodic properties. In particular, the asymptotic properties of the sequence $\left\{n_{k} x\right\}$ are similar to those of independent, identically distributed random variables; here $\{\cdot\}$ denotes fractional part. In this paper we prove that under mild assumptions on the periodic function $f$, the sequence $f\left(n_{k} x\right)$ obeys the central limit theorem and the law of the iterated logarithm after any permutation of its terms. Note that the permutational invariance of the CLT and LIL generally fails for lacunary sequences $f\left(m_{k} x\right)$ even if $\left(m_{k}\right)$ has Hadamard gaps. Our proof depends on recent deep results of Amoroso and Viada on Diophantine equations. We will also show that $\left\{n_{k} x\right\}$ satisfies a strong independence property ("interlaced mixing"), enabling one to determine the precise asymptotic behavior of permuted sums $S_{N}(\sigma)=\sum_{k=1}^{N} f\left(n_{\sigma(k)} x\right)$.
\end{abstract}

\section{INTRODUCTION}

Let $q_{1}, \ldots, q_{r}$ be a fixed set of coprime integers and let $\left(n_{k}\right)$ be the set of numbers $q_{1}^{\alpha_{1}} \cdots q_{r}^{\alpha_{r}},\left(\alpha_{i} \geq 0\right.$ integers) arranged in increasing order. Such sequences are (sometimes) called Hardy-Littlewood-Pólya sequences, and their distribution has been investigated extensively in number theory. Thue 25] showed that $n_{k+1}-n_{k} \rightarrow$ $\infty$, and this result was improved gradually until Tijdeman [26] proved that

$$
n_{k+1}-n_{k} \geq \frac{n_{k}}{\left(\log n_{k}\right)^{\alpha}}
$$

for some $\alpha>0$, i.e. the growth of $\left(n_{k}\right)$ is almost exponential. Except for the value of the constant $\alpha$, this result is best possible. Hardy-Littlewood-Pólya sequences also have remarkable probabilistic and ergodic properties. In his celebrated paper on

Received by the editors September 10, 2009.

2000 Mathematics Subject Classification. Primary 42A55, 11K60, 60F05, $60 \mathrm{~F} 15$.

Key words and phrases. Lacunary series, mixing, central limit theorem, law of the iterated logarithm, Diophantine equations.

The first author's research was supported by Austrian Science Fund Grant No. S9603-N23 and an MOEL scholarship of the Österreichisch Forschungsgemeinschaft.

The second author's research was supported by Austrian Science Fund Grant No. S9603-N23 and OTKA grants K 67961 and K 81928.

The third author's research was supported by Austrian Science Fund Grant No. S9603-N23.

(C)2011 American Mathematical Society Reverts to public domain 28 years from publication 
the Khinchin conjecture, Marstrand [15] proved that if $f$ is a bounded measurable function with period 1 , then

$$
\lim _{N \rightarrow \infty} \frac{1}{N} \sum_{k=1}^{N} f\left(n_{k} x\right)=\int_{0}^{1} f(t) d t \quad \text { a.e. }
$$

and Nair [16] showed (cf. Baker [2]) that this remains valid if instead of the boundedness of $f$ we assume only $f \in L^{1}(0,1)$. Letting $\{\cdot\}$ denote fractional part, it follows that $\left\{n_{k} x\right\}$ is not only uniformly distributed $\bmod 1$ for almost all $x$ in the sense of Weyl [27, but satisfies the "strong uniform distribution" property of Khinchin [13]. Letting

$$
D_{N}=D_{N}\left(x_{1}, \ldots, x_{N}\right):=\sup _{0 \leq a<b<1}\left|\frac{1}{N} \#\left\{k \leq N: a \leq x_{k}<b\right\}-(b-a)\right|
$$

denote the discrepancy of a sequence $\left(x_{k}\right)_{1 \leq k \leq N}$ in $(0,1)$, Philipp 18 proved, verifying a conjecture of R.C. Baker, that

$$
\frac{1}{4 \sqrt{2}} \leq \limsup _{N \rightarrow \infty} \sqrt{\frac{N}{2 \log \log N}} D_{N}\left(\left\{n_{k} x\right\}_{1 \leq k \leq N}\right) \leq C \quad \text { a.e., }
$$

with a constant $C$ depending on the generating elements of $\left(n_{k}\right)$, establishing the law of the iterated logarithm for the discrepancies of $\left\{n_{k} x\right\}$. Note that if $\left(\xi_{k}\right)$ is a sequence of independent random variables with uniform distribution over $(0,1)$, then

$$
\limsup _{N \rightarrow \infty} \sqrt{\frac{N}{2 \log \log N}} D_{N}\left(\xi_{1}, \ldots, \xi_{N}\right)=\frac{1}{2}
$$

with probability one by the Chung-Smirnov LIL (see e.g. [22], p. 504). A comparison of (1.1) and (1.2) shows that the sequence $\left\{n_{k} x\right\}$ behaves like a sequence of independent random variables. However, as Fukuyama [6] and Fukuyama and Nakata 8 showed, the limsup in (1.1) is generally different from the constant $1 / 2$ in (1.2), and it depends sensitively on the generating elements $q_{1}, \ldots, q_{r}$. For example, for $n_{k}=q^{k}, q \geq 2$, the limsup $\Sigma_{q}$ in (1.1) equals

$$
\begin{array}{ll}
\Sigma_{q}=\sqrt{42} / 9 & \text { if } q=2, \\
\Sigma_{q}=\frac{\sqrt{(q+1) q(q-2)}}{2 \sqrt{(q-1)^{3}}} & \text { if } q \geq 4 \text { is an even integer, } \\
\Sigma_{q}=\frac{\sqrt{q+1}}{2 \sqrt{q-1}} & \text { if } q \geq 3 \text { is an odd integer, }
\end{array}
$$

and if all the generating elements $q_{i}$ of $\left(n_{k}\right)$ are odd, then the limsup in (1.1) equals

$$
\frac{1}{2}\left(\prod_{i=1}^{r} \frac{q_{i}+1}{q_{i}-1}\right)^{1 / 2} .
$$

Even more surprisingly, Fukuyama [7] showed that the limsup $\Sigma$ in (1.1) is not permutation-invariant: changing the order of the $\left(n_{k}\right)$ generally changes the value of $\Sigma$. This is quite unexpected, since $\left\{n_{k} x\right\}$ are identically distributed in the sense of probability theory and the asymptotic properties of i.i.d. random variables are permutation-invariant. The purpose of this paper is to give a detailed study of the structure of $\left\{n_{k} x\right\}$ in order to explain the role of arithmetic effects and the above 
surprising deviations from i.i.d. behavior. Specifically, we will establish an "interlaced" mixing property for normed sums of $\left\{n_{k} x\right\}$, expressed by Lemmas 4 and 6 , implying that the sequence $\left\{n_{k} x\right\}$ has mixing properties after any permutation of its terms. This property is considerably stronger than standard weak dependence properties of lacunary series, which are typically valid only in the natural order of elements and will be deduced from profound Diophantine properties of $\left(n_{k}\right)$ proved recently by Amoroso and Viada 11. As a consequence, we obtain that for any permutation $\sigma: \mathbb{N} \rightarrow \mathbb{N}$ of the positive integers $\sum_{k<N} f\left(n_{\sigma(k)} x\right)$ obeys, under mild conditions on the periodic function $f$, the CLT and LIL, except that the norming sequence depends strongly on the permutation $\sigma$. Computing the norming factors in the permuted CLT and LIL is generally a difficult number theoretical problem; examples will be given below and in Section 3 .

Theorem 1. Let $f: \mathbb{R} \rightarrow \mathbb{R}$ be a measurable function satisfying the condition

$$
f(x+1)=f(x), \quad \int_{0}^{1} f(x) d x=0, \quad \operatorname{Var}_{[0,1]} f<+\infty
$$

and let $\sigma: \mathbb{N} \rightarrow \mathbb{N}$ be a permutation of $\mathbb{N}$. Assume that

$$
A_{N, M}^{2}:=\int_{0}^{1}\left(\sum_{k=M+1}^{M+N} f\left(n_{\sigma(k)} x\right)\right)^{2} d x \geq C N, \quad N \geq N_{0}, M \geq 1,
$$

for some constant $C>0$. Then letting $A_{N}=A_{N, 0}$ we have

$$
A_{N}^{-1} \sum_{k=1}^{N} f\left(n_{\sigma(k)} x\right) \rightarrow_{d} N(0,1)
$$

and

$$
\limsup _{N \rightarrow \infty} \frac{1}{\left(2 A_{N}^{2} \log \log A_{N}^{2}\right)^{1 / 2}} \sum_{k=1}^{N} f\left(n_{\sigma(k)} x\right)=1 \quad \text { a.e. }
$$

As the example $f(x)=\cos 2 \pi x-\cos 4 \pi x, n_{k}=2^{k}$ shows, assumption (1.4) cannot be omitted in Theorem 1, It is satisfied, e.g., if all Fourier coefficients of $f$ are nonnegative.

Theorem 1 shows that the asymptotic behavior of $\sum_{k=1}^{N} f\left(n_{\sigma(k)} x\right)$ is determined by the quantity

$$
A_{N}^{2}=A_{N}^{2}(f, \sigma)=\int_{0}^{1}\left(\sum_{k=1}^{N} f\left(n_{\sigma(k)} x\right)\right)^{2} d x .
$$

In the harmonic case $f(x)=\sin 2 \pi x$ we have $A_{N}(f, \sigma)=\sqrt{N / 2}$ for any $\sigma$, and thus the partial sum behavior is permutation-invariant. For trigonometric polynomials $f$ containing at least two terms the situation is different: for example, we will see that in the case $f(x)=\cos 2 \pi x+\cos 4 \pi x$ the values $\lim _{N \rightarrow \infty} A_{N}(f, \sigma) / \sqrt{N}$ for all permutations $\sigma$ fill an interval. In the case $f(x)=\{x\}-1 / 2$ we have, by a well-known identity of Landau (see [14], p. 170),

Hence in this case

$$
\int_{0}^{1} f(a x) f(b x) d x=\frac{1}{12}(a, b) /[a, b] .
$$

$$
A_{N}^{2}=\frac{1}{12} G\left(n_{\sigma(1)}, \ldots, n_{\sigma(N)}\right),
$$


where

$$
G\left(m_{1}, \ldots, m_{N}\right)=\sum_{1 \leq i \leq j \leq N} \frac{\left(m_{i}, m_{j}\right)}{\left[m_{i}, m_{j}\right]} .
$$

Here $(a, b)$ and $[a, b]$ denote the greatest common divisor, resp. least common multiple of $a$ and $b$. The function $G$ plays an important role in the metric theory of Diophantine approximation; see the profound paper of Gál [10 for more information on this point. Clearly, $G\left(n_{\sigma(1)}, \ldots, n_{\sigma(N)}\right) \geq N$, and from the proof of Lemma 2.2 of Philipp [18] it is easily seen that

$$
G\left(n_{\sigma(1)}, \ldots, n_{\sigma(N)}\right) \ll N .
$$

Here, and in the sequel, « means the same as the $O$ notation. In the case of the identity permutation $\sigma$ the value of $\lim _{N \rightarrow \infty} N^{-1} G\left(n_{1}, \ldots, n_{N}\right)$ was computed by Fukuyama and Petit [9]; in Section 3 we will determine the class of limits $\lim _{N \rightarrow \infty} N^{-1} G\left(n_{\sigma(1)}, \ldots, n_{\sigma(N)}\right)$ in the case $n_{k}=2^{k}$.

Corollary. Let $f: \mathbb{R} \rightarrow \mathbb{R}$ be a measurable function satisfying (1.3) and assume that the Fourier coefficients of $f$ are nonnegative. Let $\sigma$ be a permutation of $\mathbb{N}$. Then $N^{-1 / 2} \sum_{k=1}^{N} f\left(n_{\sigma(k)} x\right)$ has a nondegenerate limit distribution iff

$$
\gamma^{2}=\lim _{N \rightarrow \infty} N^{-1} \int_{0}^{1}\left(\sum_{k=1}^{n} f\left(n_{\sigma(k)} x\right)\right)^{2} d x>0
$$

exists, and then

$$
N^{-1 / 2} \sum_{k=1}^{N} f\left(n_{\sigma(k)} x\right) \rightarrow_{d} N\left(0, \gamma^{2}\right) .
$$

Also, if condition (1.8) is satisfied, then

$$
\limsup _{N \rightarrow \infty} \frac{1}{\sqrt{2 N \log \log N}} \sum_{k=1}^{N} f\left(n_{\sigma(k)} x\right)=\gamma \quad \text { a.e. }
$$

As mentioned, for the original, unpermuted sequence $\left(n_{k}\right)$, the value of $\gamma=\gamma_{f}$ in (1.8) was computed in [9. Given an $f$ satisfying condition (1.3), let $\Gamma_{f}$ denote the set of limiting variances in (1.8) belonging to all permutations $\sigma$. (Note that the limit does not always exist.) Despite the simple description of $\Gamma_{f}$ above, it seems a difficult problem to determine this set explicitly. In Section 3 we will prove that for $n_{k}=2^{k}$ and functions $f$ with nonnegative Fourier coefficients, $\Gamma_{f}$ is identical with the interval determined by $\|f\|^{2}$ and $\gamma_{f}^{2}$. For $f$ having also negative coefficients this is false, as an example in Section 3 will show.

In conclusion, we note that the results of our paper are due to the special arithmetic properties of Hardy-Littlewood-Pólya sequences and they become generally false for sequences $f\left(m_{k} x\right)$ even if $\left(m_{k}\right)$ satisfies the Hadamard gap condition $m_{k+1} / m_{k} \geq q>1(k=1,2, \ldots)$. At the end of Section 3 we will construct a Hadamard lacunary sequence $\left(m_{k}\right)$ such that $f\left(m_{k} x\right)$ satisfies the CLT and LIL for all periodic Lipschitz functions $f$, but for a suitable permutation $\sigma: \mathbb{N} \rightarrow \mathbb{N}$ and a trigonometric polynomial $f$, the normed partial sums of $f\left(m_{\sigma(k)} x\right)$ have a nongaussian limit distribution. 


\section{AN INTERLACED MIXING PROPERTY}

The crucial tool in proving Theorem 1 is a recent deep bound for the number of solutions $\left(k_{1}, \ldots, k_{p}\right)$ of the Diophantine equation

$$
a_{1} n_{k_{1}}+\cdots+a_{p} n_{k_{p}}=b
$$

where $a_{1}, \ldots, a_{p}, b$ are nonzero integers. Call a solution $\left(k_{1}, \ldots, k_{p}\right)$ of (2.1) nondegenerate if no subsum of the sum on the left hand side equals 0 . Amoroso and Viada [1] proved the following result, improving the quantitative subspace theorem of Schmidt [20] (cf. also Evertse et al. [5]).

Lemma 1. For any nonzero integers $a_{1}, \ldots, a_{p}, b$ the number of nondegenerate solutions of (2.1) is at most $\exp \left(c p^{6}\right)$, where $c$ is a constant depending only on the number of generators of $\left(n_{k}\right)$.

Dividing by $b$ in (2.1), we can assume, alternatively, that $a_{1}, \ldots, a_{p}$ are nonzero rational numbers and $b=1$; this is the formulation used in [1].

For the rest of the paper, $C$ will denote positive constants, possibly different at different places, depending (at most) on $f$ and $\left(n_{k}\right)$. Similarly, the constants implied by $O$ and by the equivalent relation $\ll$ will depend (at most) on $f$ and $\left(n_{k}\right)$.

Most results of this paper are probabilistic statements on the sequence $\left(f\left(n_{k} x\right)\right)_{k \geq 1}$, and we will use probabilistic terminology. The underlying probability space for our sequence is the interval $[0,1]$, equipped with Borel sets and the Lebesgue measure; we will denote probability and expectation in this space by $\mathbb{P}$ and $\mathbb{E}$.

Given any finite set $I$ of positive integers, set

$$
S_{I}=\sum_{k \in I} f\left(n_{k} x\right), \quad \sigma_{I}=\left(\mathbb{E} S_{I}^{2}\right)^{1 / 2} .
$$

From Lemma 1 we deduce

Lemma 2. Assume the conditions of Theorem 1 and let $I$ be a set of positive integers with cardinality $N$. Then we have for any integer $p \geq 3$

$$
\mathbb{E} S_{I}^{p}= \begin{cases}\frac{p !}{(p / 2) !} 2^{-p / 2} \sigma_{I}^{p}+O\left(T_{N}\right) & \text { if } p \text { is even, } \\ O\left(T_{N}\right) & \text { if } p \text { is odd }\end{cases}
$$

where

$$
T_{N}=\exp \left(C p^{7}\right) N^{(p-1) / 2}(\log N)^{p} .
$$

Proof. Let $C_{p}=\exp \left(c p^{6}\right)$ be the constant in Lemma 1. We first note that

$$
\sigma_{I} \leq K\|f\|^{1 / 2}|I|^{1 / 2},
$$

where $K$ is a constant depending only on the generating elements of $\left(n_{k}\right)$. This relation is implicit in the proof of Lemma 2.2 of Philipp [18. Next we observe that for any fixed $p \geq 3$ and any fixed nonzero coefficients $a_{1}, \ldots, a_{p}$, the number of nondegenerate solutions of (2.1) such that $b=0$ and $k_{1}, \ldots, k_{p} \in I$ is at most $C_{p-1} N$. Indeed, the number of choices for $k_{p}$ is at most $N$, and thus taking $a_{p} n_{k_{p}}$ to the right hand side and applying Lemma 1, our claim follows. 
Without loss of generality we may assume that $f$ is an even function and that $\|f\|_{\infty} \leq 1, \operatorname{Var}_{[0,1]} f \leq 1$; the proof in the general case is the same. (Here and in the sequel, $\|\cdot\|_{p}$ denotes the $L_{p}$ norm; for $p=2$ we simply write $\|\cdot\|$.) Let

$$
f \sim \sum_{j=1}^{\infty} a_{j} \cos 2 \pi j x
$$

be the Fourier series of $f$. $\operatorname{Var}_{[0,1]} f \leq 1$ implies (see Zygmund [28, p. 48])

$$
\left|a_{j}\right| \leq j^{-1}
$$

and, writing

$$
g(x)=\sum_{j=1}^{N^{2 p}} a_{j} \cos 2 \pi j x, \quad r(x)=f(x)-g(x),
$$

we have

$$
\|g\|_{\infty} \leq \operatorname{Var}_{[0,1]} f+\|f\|_{\infty} \leq 2, \quad\|r\|_{\infty} \leq\|f\|_{\infty}+\|g\|_{\infty} \leq 3 .
$$

For any positive integer $n,(2.3)$ yields

$$
\|r(n x)\|^{2}=\|r(x)\|^{2}=\frac{1}{2} \sum_{j=N^{2 p}+1}^{\infty} a_{j}^{2} \leq N^{-2 p} .
$$

By Minkowski's inequality,

$$
\left\|S_{I}\right\|_{p} \leq\left\|\sum_{k \in I} g\left(n_{k} x\right)\right\|_{p}+\left\|\sum_{k \in I} r\left(n_{k} x\right)\right\|_{p}
$$

and

$$
\left\|\sum_{k \in I} r\left(n_{k} x\right)\right\|_{p} \leq 3 \sum_{k \in I}\left\|r\left(n_{k} x\right) / 3\right\|_{p} \leq 3 \sum_{k \in I}\left\|r\left(n_{k} x\right) / 3\right\|^{2 / p} \leq 3 \sum_{k \in I} N^{-2} \leq 3 .
$$

By expanding and using elementary properties of the trigonometric functions we get

$$
\begin{aligned}
& \mathbb{E}\left(\sum_{k \in I} g\left(n_{k} x\right)\right)^{p} \\
& =2^{-p} \sum_{1 \leq j_{1}, \ldots, j_{p} \leq N^{2 p}} a_{j_{1}} \cdots a_{j_{p}} \sum_{k_{1}, \ldots, k_{p} \in I} \mathbb{I}\left\{ \pm j_{1} n_{k_{1}} \pm \ldots \pm j_{p} n_{k_{p}}=0\right\}
\end{aligned}
$$

with all possibilities of the signs \pm within the indicator function. Assume that $j_{1}, \ldots, j_{p}$ and the signs \pm are fixed, and consider a solution of $\pm j_{1} n_{k_{1}} \pm \cdots \pm j_{p} n_{k_{p}}=$ 0 . Then the set $\{1,2, \ldots, p\}$ can be split into disjoint sets $A_{1}, \ldots, A_{l}$ such that for each such set $A$ we have $\sum_{i \in A} \pm j_{i} n_{k_{i}}=0$ and no further subsums of these sums are equal to 0 . By the monotonicity of $C_{p}$ and the remark at the beginning of the proof, for each $A$ with $|A| \geq 3$ the number of solutions is $\leq C_{|A|-1} N \leq C_{p-1} N$; trivially, for $|A|=2$ the number of solutions is at most $N$. Thus if $s_{i}=\left|A_{i}\right| \quad(1 \leq i \leq p)$ 
denotes the cardinality of $A_{i}$, the number of solutions of $\pm j_{1} n_{k_{1}} \pm \cdots \pm j_{p} n_{k_{p}}=0$ admitting such a decomposition with fixed $A_{1}, \ldots, A_{l}$ is at most

$$
\begin{aligned}
& \prod_{\left\{i: s_{i} \geq 3\right\}} C_{p-1} N \prod_{\left\{i: s_{i}=2\right\}} N \leq\left(C_{p-1} N\right)^{\sum_{\left\{i: s_{i} \geq 3\right\}} 1+\sum_{\left\{i: s_{i}=2\right\}} 1} \\
& \leq\left(C_{p-1} N\right)^{\frac{1}{3} \sum_{\left\{i: s_{i} \geq 3\right\}} s_{i}+\frac{1}{2} \sum_{\left\{i: s_{i}=2\right\}} s_{i}}=\left(C_{p-1} N\right)^{\frac{1}{3} \sum_{\left\{i: s_{i} \geq 3\right\}} s_{i}+\frac{1}{2}\left(p-\sum_{\left\{i: s_{i} \geq 3\right\}} s_{i}\right)} \\
& =\left(C_{p-1} N\right)^{\frac{p}{2}-\frac{1}{6} \sum_{\left\{i: s_{i} \geq 3\right\}} s_{i}} .
\end{aligned}
$$

If there is at least one $i$ with $s_{i} \geq 3$, then the last exponent is at most $(p-1) / 2$, and since the number of partitions of the set $\{1, \ldots, p\}$ into disjoint subsets is at most $p ! 2^{p}$, we see that the number of solutions of $\pm j_{1} n_{k_{1}} \pm \cdots \pm j_{p} n_{k_{p}}=0$, where at least one of the sets $A_{i}$ has cardinality $\geq 3$, is at most $p ! 2^{p}\left(C_{p-1} N\right)^{(p-1) / 2}$. If $p$ is odd, there are no other solutions, and thus using (2.3) the inner sum in (2.5) is at most $p ! 2^{p}\left(C_{p-1} N\right)^{(p-1) / 2}$, and consequently, taking into account the $2^{p}$ choices for the signs \pm 1 ,

$$
\begin{aligned}
& \left|\mathbb{E}\left(\sum_{k \in I} g\left(n_{k} x\right)\right)^{p}\right| \\
& \leq p ! 2^{p}\left(C_{p-1} N\right)^{(p-1) / 2} 2^{p} \sum_{1 \leq j_{1}, \ldots, j_{p} \leq N^{2 p}}\left|a_{j_{1}} \cdots a_{j_{p}}\right| \ll \exp \left(C p^{7}\right) N^{(p-1) / 2}(\log N)^{p} .
\end{aligned}
$$

If $p$ is even, there are also solutions where each $A$ has cardinality 2. Clearly, these are trivial solutions and, as we will see, their contribution in $\mathbb{E} S_{I}^{p}$ dominates the previously considered nontrivial solutions. Indeed, the contribution of the terms in (2.5), where $A_{1}=\{1,2\}, A_{2}=\{3,4\}, \ldots$, is

$$
\begin{aligned}
& \left(\frac{1}{4} \sum_{1 \leq i, j \leq N^{2 p}} \sum_{k, \ell \in I} a_{i} a_{j} \mathbb{I}\left\{ \pm i n_{k} \pm j n_{\ell}=0\right\}\right)^{p / 2}=\left(\mathbb{E}\left(\sum_{k \in I} g\left(n_{k} x\right)\right)^{2}\right)^{p / 2} \\
& =\left\|\sum_{k \in I} g\left(n_{k} x\right)\right\|^{p}=\left\|S_{I}-\sum_{k \in I} r\left(n_{k} x\right)\right\|^{p}=\left(\sigma_{I}+O(1)\right)^{p} \\
& =\sigma_{I}^{p}+p\left(\sigma_{I}+O(1)\right)^{p-1} O(1) \\
& =\sigma_{I}^{p}+O\left(p 2^{p-2}\right)\left(\sigma_{I}^{p-1}+O(1)^{p-1}\right) \\
& =\sigma_{I}^{p}+O\left(2^{p^{2}}\right) N^{(p-1) / 2}
\end{aligned}
$$

using the mean value theorem and the relation

$$
\left(\sum_{j=1}^{m} x_{j}\right)^{\alpha} \leq \max \left(1, m^{\alpha-1}\right) \sum_{j=1}^{m} x_{j}^{\alpha} \quad\left(\alpha>0, x_{i} \geq 0\right) .
$$

Here the constants implied by the $O$ are absolute. Since the splitting of $\{1,2, \ldots, p\}$ into pairs can be done in $\frac{p !}{(p / 2) !} 2^{-p / 2}$ different ways, we proved that

$$
\mathbb{E}\left(\sum_{k \in I} g\left(n_{k} x\right)\right)^{p}=\left\{\begin{array}{l}
\frac{p !}{(p / 2) !} 2^{-\frac{p}{2}} \sigma_{I}^{p}+O\left(T_{N}^{*}\right) \\
O\left(T_{N}^{*}\right)
\end{array}\right.
$$


according to whether $p$ is even or odd; here

$$
T_{N}^{*}=\exp \left(C p^{7}\right) N^{(p-1) / 2}(\log N)^{p} .
$$

Now, letting $G_{I}=\sum_{k \in I} g\left(n_{k} x\right)$ and $R_{I}=\sum_{k \in I} r\left(n_{k} x\right)$, we get, using the mean value theorem, Hölder's inequality and (2.7),

$$
\begin{aligned}
& \left|\mathbb{E} S_{I}^{p}-\mathbb{E} G_{I}^{p}\right| \\
& \leq \mathbb{E}\left|\left(G_{I}+R_{I}\right)^{p}-G_{I}^{p}\right|=\mathbb{E}\left|p R_{I}\left(G_{I}+\theta R_{I}\right)^{p-1}\right| \\
& \leq p\left\|R_{I}\right\|_{p}\left\|\left(G_{I}+\theta R_{I}\right)^{p-1}\right\|_{p /(p-1)}=p\left\|R_{I}\right\|_{p}\left\|G_{I}+\theta R_{I}\right\|_{p}^{p-1} \\
& \leq 3 p\left(\left\|G_{I}\right\|_{p}+3\right)^{p-1} \leq 3 p 2^{p-2}\left(\left\|G_{I}\right\|_{p}^{p-1}+3^{p-1}\right),
\end{aligned}
$$

for some $0 \leq \theta=\theta(x) \leq 1$. For even $p$ we get from (2.8), together with (2.7) with $\alpha=1 / p$, that

$$
\left\|G_{I}\right\|_{p} \ll p \sigma_{I}+\exp \left(C p^{6}\right) \sqrt{N} \log N
$$

For $p$ odd, we get the same bound, since $\left\|G_{I}\right\|_{p} \leq\left\|G_{I}\right\|_{p+1}$. Thus for any $p \geq 3$ we get from (2.9)

$$
\begin{aligned}
\mathbb{E} S_{I}^{p} & \leq \mathbb{E} G_{I}^{p}+3 p 2^{p-2}\left(\left\|G_{I}\right\|_{p}^{p-1}+3^{p-1}\right) \\
& \leq \mathbb{E} G_{I}^{p}+3 p 2^{p-2}\left[\left(p \sigma_{I} \exp \left(C p^{6}\right)\right)^{p-1}+3^{p-1}\right] O(1)^{p-1} \\
& \leq E G_{I}^{p}+\exp \left(C p^{7}\right)(C \sqrt{N})^{p-1},
\end{aligned}
$$

completing the proof of Lemma 2 .

Lemma 3. Let

$$
f=\sum_{k=1}^{d}\left(a_{k} \cos 2 \pi k x+b_{k} \sin 2 \pi k x\right)
$$

be a trigonometric polynomial and let $I, J$ be disjoint sets of positive integers with cardinality $M$ and $N$, respectively, where $M / N \leq C$ with a sufficiently small constant $0<C<1$. Assume $\sigma_{I} \gg|I|^{1 / 2}$ and $\sigma_{J} \gg|J|^{1 / 2}$. Then for any integers $p \geq 2$ and $q \geq 2$ we have

$$
\begin{aligned}
& \mathbb{E}\left(S_{I} / \sigma_{I}\right)^{p}\left(S_{J} / \sigma_{J}\right)^{q} \\
& = \begin{cases}\frac{p !}{(p / 2) ! 2^{p / 2}} \frac{q !}{(q / 2) ! 2^{q / 2}}+O\left(T_{M, N}\right) & \text { if } p, q \text { are even }, \\
O\left(T_{M, N}\right) & \text { otherwise, }\end{cases}
\end{aligned}
$$

where

$$
T_{M, N}=C_{p+q}^{p+q}\left(M^{-1 / 2}+(M / N)^{1 / 2}\right),
$$

and $C_{p}=\exp \left(c p^{6}\right)$ is the constant in Lemma 1 .

Proof. To simplify the formulas, we assume again that $f$ is a cosine polynomial, i.e.

$$
f(x)=\sum_{j=1}^{d} a_{j} \cos 2 \pi j x .
$$


The general case requires only trivial changes. Clearly

$$
\begin{aligned}
& S_{I}^{p} S_{J}^{q}=\frac{1}{2^{p+q}} \sum_{\substack{1 \leq j_{1}, \ldots, j_{p+q} \leq d \\
k_{j_{1}} \cdots a_{j_{p+q}}}} a_{\substack{k_{1}, \ldots, k_{p} \in I \\
k_{p+1}, \ldots, k_{p+q} \in J}} \cos 2 \pi\left( \pm j_{1} n_{k_{1}} \pm \cdots \pm j_{p+q} n_{k_{p+q}}\right) x,
\end{aligned}
$$

and thus

$$
\begin{aligned}
\mathbb{E} S_{I}^{p} S_{J}^{q} & =\frac{1}{2^{p+q}} \sum_{\substack{1 \leq j_{1}, \ldots, j_{p+q} \leq d \\
j_{j_{1}} \ldots a_{j_{p+q}}}} \sum \sum_{\substack{k_{1}, \ldots, k_{p} \in I \\
k_{p+1}, \ldots, k_{p+q} \in J}} I\left\{ \pm j_{1} n_{k_{1}} \pm \cdots \pm j_{p+q} n_{k_{p+q}}=0\right\} .
\end{aligned}
$$

Assume that $j_{1}, \ldots, j_{p+q}$ and the signs \pm are fixed, and consider a solution of

$$
\pm j_{1} n_{k_{1}} \pm \cdots \pm j_{p+q} n_{k_{p+q}}=0 .
$$

Clearly, the set $\{1,2, \ldots, p+q\}$ can be split into disjoint sets $A_{1}, \ldots, A_{\ell}$ such that for each such set $A$ we have $\sum_{i \in A} \pm j_{i} n_{k_{i}}=0$ and no further subsums of these sums are equal to 0 . Call a set $A$ type 1 or type 2 according to whether $A$ intersects $\{1,2, \ldots, p\}$ or $A \subseteq\{p+1, \ldots, p+q\}$. Similarly as in the proof of Lemma 2, the number of solutions of the equation $\sum_{i \in A} \pm j_{i} n_{k_{i}}=0$ is at most $C_{p+q-1} M$ or $C_{p+q-1} N$ according to whether $A$ is of type 1 or type 2 . Thus the number of solutions of (2.12) belonging to a fixed decomposition $\left\{A_{1}, \ldots, A_{\ell}\right\}$ is at most

$$
\left(C_{p+q-1} M\right)^{R}\left(C_{p+q-1} N\right)^{S},
$$

where $R$ and $S$ denote, respectively, the number of $A_{i}$ 's with type 1 and type 2 . Let $R^{*}$ and $S^{*}$ denote the total cardinality of sets of type 1 and type 2 . Then $R=R^{*} / 2$ or $R \leq\left(R^{*}-1\right) / 2$ according to whether all sets of type 1 have cardinality 2 or at least one of them has cardinality $\geq 3$. A similar statement holds for sets of type 2 , and thus if there exists at least one set $A_{i}$ with $\left|A_{i}\right| \geq 3$, the expression in (2.13) can be estimated as follows, also using $R^{*}+S^{*}=p+q, S^{*} \leq q$ :

$$
\begin{aligned}
\left(C_{p+q-1} M\right)^{R}\left(C_{p+q-1} N\right)^{S} & \leq\left(C_{p+q-1} M\right)^{R^{*} / 2}\left(C_{p+q-1} N\right)^{S^{*} / 2}\left(C_{p+q-1} M\right)^{-1 / 2} \\
& =\left(C_{p+q-1} M\right)^{\left(p+q-S^{*}\right) / 2}\left(C_{p+q-1} N\right)^{S^{*} / 2}\left(C_{p+q-1} M\right)^{-1 / 2} \\
& =\left(C_{p+q-1} M\right)^{(p+q) / 2}(N / M)^{S^{*} / 2}\left(C_{p+q-1} M\right)^{-1 / 2} \\
& \leq\left(C_{p+q-1} M\right)^{(p+q) / 2}(N / M)^{q / 2}\left(C_{p+q-1} M\right)^{-1 / 2} \\
& \leq C_{p+q}^{(p+q) / 2} M^{p / 2} N^{q / 2} M^{-1 / 2} \\
& \ll C^{p+q} C_{p+q}^{(p+q) / 2} \sigma_{I}^{p} \sigma_{J}^{q} M^{-1 / 2}
\end{aligned}
$$

where in the last step we used (1.4). Since the total number of decompositions of the set $\{1,2, \ldots, p+q\}$ into subsets is $\leq(p+q) ! 2^{p+q} \ll 2^{(p+q)^{2}}$, it follows that the contribution of those solutions of (2.12) in (2.11), where $\left|A_{i}\right| \geq 3$ for at least one set $A_{i}$, is

$$
\ll 2^{(p+q)^{2}}(\log d)^{p+q} C_{p+q}^{(p+q) / 2} M^{-1 / 2} \sigma_{I}^{p} \sigma_{J}^{q} .
$$

We now turn to the contribution of those solutions of (2.12) where all sets $A_{1}, \ldots, A_{\ell}$ have cardinality 2 . This can happen only if $p+q$ is even and then 
$\ell=(p+q) / 2$. Fixing $A_{1}, \ldots, A_{\ell}$, the sum of the corresponding terms in (2.11) can be written as

$$
2^{-(p+q)} \sum_{1 \leq j_{1}, \ldots, j_{p+q} \leq d} a_{j_{1}} \cdots a_{j_{p+q}} I\left\{\sum_{i \in A_{1}} \pm j_{i} n_{k_{i}}=0\right\} \cdots I\left\{\sum_{i \in A_{(p+q) / 2}} \pm j_{i} n_{k_{i}}=0\right\}
$$

and this is the product of $(p+q) / 2$ such sums belonging to $A_{1}, \ldots, A_{(p+q) / 2}$. For an $A_{i} \subseteq\{1, \ldots, p\}$ we get

$$
\frac{1}{4} \sum_{\substack{1 \leq i, j \leq d \\ k_{i}, k_{j} \in I}} a_{i} a_{j} I\left\{ \pm i n_{k_{i}} \pm j n_{k_{j}}=0\right\}=E S_{I}^{2}=\sigma_{I}^{2} .
$$

Similarly, for any $A_{i} \subseteq\{p+1, \ldots, p+q\}$ the corresponding sum equals $E S_{J}^{2}=\sigma_{J}^{2}$. Finally, if a set $A_{i}$ is "mixed", i.e. if one of its elements is in $\{1, \ldots, p\}$ and the other is in $\{p+1, \ldots, p+q\}$, then we get $E S_{I} S_{J}:=\sigma_{I, J}$ (cf. (2.11) with $p=q=1$ ). Thus, if we have $t_{1}$ sets $A_{i} \subseteq\{1, \ldots, p\}, t_{2}$ sets $A_{i} \subseteq\{p+1, \ldots, p+q\}$ and $t_{3}$ "mixed" sets, we get $\sigma_{I}^{2 t_{1}} \sigma_{J}^{2 t_{2}} \sigma_{I, J}^{t_{3}}$. Clearly $t_{3}=0$ can occur only if $p$ and $q$ are both even and then $t_{1}=p / 2, t_{2}=q / 2$, i.e. we get $\sigma_{I}^{p} \sigma_{J}^{q}$ which, taking into account the fact that $\{1,2, \ldots, p\}$ can be split into 2-element subsets in $\frac{p !}{(p / 2) !} 2^{-p / 2}$ different ways, gives the contribution

$$
\frac{p !}{(p / 2) ! 2^{p / 2}} \frac{q !}{(q / 2) ! 2^{q / 2}} \sigma_{I}^{p} \sigma_{J}^{q}
$$

Assume now that $t_{3}=s, 1 \leq s \leq p \wedge q$. Then $t_{1}=(p-s) / 2, t_{2}=(q-s) / 2$; clearly if $p$ and $q$ are both even, then $s$ can be $0,2,4, \ldots$ and if $p$ and $q$ are both odd, then $s$ can be $1,3,5, \ldots$. Thus the contribution in this case is

$$
\sigma_{I}^{p-s} \sigma_{J}^{q-s} \sigma_{I, J}^{s}
$$

From

$$
\sigma_{I, J}=\frac{1}{4} \sum_{\substack{1 \leq i, j \leq d \\ k \in I, \ell \in J}} a_{i} a_{j} I\left\{ \pm i n_{k} \pm j n_{\ell}=0\right\}
$$

we see that $\sigma_{I, J} \ll(|I| \wedge|J|)=M$, and thus dividing with $\sigma_{I}^{p} \sigma_{J}^{q}$ and summing for $s,(2.14)$ yields, again using (1.4),

$$
\begin{aligned}
\sum_{s \geq 1} \sigma_{I}^{-s} \sigma_{J}^{-s} \sigma_{I, J}^{s} & \leq \sum_{s \geq 1} C^{2 s}(M N)^{-s / 2} M^{s} \\
& =\sum_{s \geq 1} C^{2 s}(M / N)^{s / 2} \ll(M / N)^{1 / 2},
\end{aligned}
$$

provided $C$ is small enough.

Lemma 4. Under the conditions of Lemma 3 we have for any $0<\delta<1$

$$
\begin{aligned}
& \left|\mathbb{E}\left(\exp \left(i t S_{I} / \sigma_{I}+i s S_{J} / \sigma_{J}\right)\right)-e^{-\left(t^{2}+s^{2}\right) / 2}\right| \\
& \ll e^{-C(\log M)^{\delta}}+e^{C(\log M)^{7 \delta}}\left(M^{-1 / 2}+\sqrt{M / N}\right)
\end{aligned}
$$

for $|t|,|s| \leq \frac{1}{4}(\log M)^{\delta / 2}$. 
Lemma 4 (and also Lemma 6 below) show that the random variables $S_{I} / \sigma_{I}$ and $S_{J} / \sigma_{J}$ are asymptotically independent if $|I| \rightarrow \infty,|J| \rightarrow \infty$, and $|I| /|J| \rightarrow 0$. Note that $I$ and $J$ are arbitrary disjoint subsets of $\mathbb{N}$ : they do not have to be intervals or be separated by some number $x \in \mathbb{R}$; they can also be "interlaced". Thus $\left\{n_{k} x\right\}$ obeys an "interlaced" mixing condition, an unusually strong near independence property introduced by Bradley [3. Note that this property is permutationinvariant, explaining the permutation-invariance of the CLT and LIL in Theorem 1.

It is easy to extend Lemma 4 for the joint characteristic function of normed sums $S_{I_{1}} / \sigma_{I_{1}}, \ldots, S_{I_{d}} / \sigma_{I_{d}}$ of $d$ disjoint blocks $I_{1}, \ldots, I_{d}, d \geq 3$. Since, however, the standard mixing conditions such as $\alpha$-mixing, $\beta$-mixing, etc. involve pairs of events and the present formulation will suffice for the CLT and LIL for $f\left(n_{\sigma(k)} x\right)$, we will consider only the case $d=2$.

Proof. Using $\left|e^{i x}-\sum_{p=0}^{k-1} \frac{(i x)^{p}}{p !}\right| \leq \frac{|x|^{k}}{k !}$, valid for any $x \in \mathbb{R}, k \geq 1$, we get for any $L \geq 1$

$$
\begin{aligned}
\exp \left(i t S_{I} / \sigma_{I}\right)= & \sum_{p=0}^{L-1} \frac{(i t)^{p}}{p !}\left(S_{I} / \sigma_{I}\right)^{p} \\
& +\theta_{L}(t, x, I) \frac{|t|^{L}}{L !}\left|S_{I} / \sigma_{I}\right|^{L} \\
= & : U_{L}(t, x, I)+\theta_{L}(t, x, I) \frac{|t|^{L}}{L !}\left|S_{I} / \sigma_{I}\right|^{L},
\end{aligned}
$$

where $\left|\theta_{L}(t, x, I)\right| \leq 1$. Writing a similar expansion for $\exp \left(i s S_{J} / \sigma_{J}\right)$ and multiplying, we get

$$
\begin{aligned}
\mathbb{E} & \left(\exp \left(i t S_{I} / \sigma_{I}+i s S_{J} / \sigma_{J}\right)\right) \\
= & \mathbb{E}\left(U_{L}(t, x, I) U_{L}(s, x, J)\right)+\mathbb{E}\left(U_{L}(t, x, I) \theta_{L}(s, x, J) \frac{|s|^{L}}{L !}\left|S_{J} / \sigma_{J}\right|^{L}\right) \\
& +\mathbb{E}\left(U_{L}(s, x, J) \theta_{L}(t, x, I) \frac{|t|^{L}}{L !}\left|S_{I} / \sigma_{I}\right|^{L}\right) \\
& +\mathbb{E}\left(\frac{|t|^{L}}{L !} \frac{|s|^{L}}{L !}\left|S_{I} / \sigma_{I}\right|^{L}\left|S_{J} / \sigma_{J}\right|^{L} \theta_{L}(t, x, I) \theta_{L}(s, x, J)\right) \\
= & I_{1}+I_{2}+I_{3}+I_{4} .
\end{aligned}
$$

We estimate $I_{1}, I_{2}, I_{3}, I_{4}$ separately. We choose $L=2\left[(\log M)^{\delta}\right]$ and use Lemma 3 to get

$$
\begin{aligned}
I_{1}= & \sum_{\substack{p, q=0 \\
p, q \text { even }}}^{L-1} \frac{(i t)^{p}}{(p / 2) ! 2^{p / 2}} \frac{(i s)^{q}}{(q / 2) ! 2^{q / 2}} \\
& +O(1) \sum_{p, q=0}^{L-1} \frac{|t|^{p}}{p !} \frac{|s|^{q}}{q !} C_{2 L}^{2 L}\left(M^{-1 / 2}+(M / N)^{1 / 2}\right) \\
= & : I_{1,1}+I_{1,2} .
\end{aligned}
$$


Here

$$
\begin{aligned}
& e^{-\left(t^{2}+s^{2}\right) / 2}-I_{1,1} \\
& =\left(\sum_{\substack{p=0 \\
p \text { even }}}^{L-1} \frac{(i t)^{p}}{(p / 2) ! 2^{p / 2}}\right)\left(\sum_{\substack{q=L \\
q \text { even }}}^{\infty} \frac{(i s)^{q}}{(q / 2) ! 2^{q / 2}}\right)+\left(\sum_{\substack{p=L \\
p \text { even }}}^{\infty} \frac{(i t)^{p}}{(p / 2) ! 2^{p / 2}}\right)\left(\sum_{\substack{q=0 \\
q \text { even }}}^{\infty} \frac{(i s)^{q}}{(q / 2) ! 2^{s / 2}}\right) .
\end{aligned}
$$

Using $n ! \geq(n / 3)^{n}$ and $t^{2} \leq L / 24 \leq p / 24$ we get

$$
\begin{aligned}
\left|\sum_{\substack{p=L \\
p \text { even }}}^{\infty} \frac{(i t)^{p}}{(p / 2) ! 2^{p / 2}}\right| & \leq \sum_{p=L}^{\infty} \frac{|t|^{p}}{(p / 3)^{p / 2}}=\sum_{p=L}^{\infty}\left(\frac{t^{2}}{p / 3}\right)^{p / 2} \\
& \leq \sum_{p=L}^{\infty}\left(\frac{1}{4}\right)^{p / 2} \leq 2 \cdot 2^{-L} \leq 8 e^{-(\log M)^{\delta}}
\end{aligned}
$$

and similarly

$$
\left|\sum_{\substack{q=L \\ q \text { even }}}^{\infty} \frac{(i s)^{q}}{(q / 2) ! 2^{q / 2}}\right| \ll e^{-(\log M)^{\delta}} .
$$

Thus

$$
\left|\sum_{\substack{p=0 \\ p \text { even }}}^{L-1} \frac{(i t)^{p}}{(p / 2) ! 2^{p / 2}}\right| \leq e^{-t^{2} / 2}+\left|\sum_{\substack{p=L \\ p \text { even }}}^{\infty} \frac{(i t)^{p}}{(p / 2) ! 2^{p / 2}}\right| \leq 9,
$$

and a similar estimate holds for

$$
\sum_{\substack{q=0 \\ q \text { even }}}^{L-1} \frac{(i s)^{q}}{(q / 2) ! 2^{q / 2}} .
$$

Consequently,

$$
\left|I_{1,1}-e^{-\left(t^{2}+s^{2}\right) / 2}\right| \ll e^{-(\log M)^{\delta}}
$$

On the other hand,

$$
\begin{aligned}
\left|I_{1,2}\right| & \ll\left(\sum_{p=0}^{\infty} \frac{|t|^{p}}{p !}\right)\left(\sum_{q=0}^{\infty} \frac{|s|^{q}}{q !}\right) C_{2 L}^{2 L}\left(M^{-1 / 2}+(M / N)^{1 / 2}\right) \\
& \ll e^{|t|+|s|} e^{C(2 L)^{7}}\left(M^{-1 / 2}+(M / N)^{1 / 2}\right) \\
& \ll e^{C(\log M)^{7 \delta}}\left(M^{-1 / 2}+(M / N)^{1 / 2}\right) .
\end{aligned}
$$

Thus we proved

$$
\left|I_{1}-e^{-\left(t^{2}+s^{2}\right) / 2}\right| \ll e^{-C(\log M)^{\delta}}+e^{C(\log M)^{7 \delta}}\left(M^{-1 / 2}+(M / N)^{1 / 2}\right) .
$$


Next we estimate $I_{4}$. Using Lemma 3 and $t^{2} \leq L / 24$ we get, since $L$ is even,

$$
\begin{aligned}
I_{4} \leq & \frac{|t|^{L}}{L !} \frac{|s|^{L}}{L !} \mathbb{E}\left|S_{I} / \sigma_{I}\right|^{L}\left|S_{J} / \sigma_{J}\right|^{L} \\
& \ll \frac{|t|^{L}}{L !} \frac{|s|^{L}}{L !}\left[\left(\frac{L !}{(L / 2) ! 2^{L / 2}}\right)^{2}+C_{2 L}^{2 L}\left(M^{-1 / 2}+(M / N)^{1 / 2}\right)\right] \\
& \ll \frac{|t|^{L}|s|^{L}}{((L / 2) !)^{2}}+\frac{|t|^{L}|s|^{L}}{(L !)^{2}} C_{2 L}^{2 L}\left(M^{-1 / 2}+(M / N)^{1 / 2}\right) \\
& \ll\left(\frac{t^{2}}{L / 6}\right)^{L / 2}\left(\frac{s^{2}}{L / 6}\right)^{L / 2} \\
& +\left(\frac{t^{2}}{L / 6}\right)^{L / 2}\left(\frac{s^{2}}{L / 6}\right)^{L / 2} e^{C(2 L)^{7}}\left(M^{-1 / 2}+(M / N)^{1 / 2}\right) \\
& \ll 4^{-L}+4^{-L} e^{C(2 L)^{7}}\left(M^{-1 / 2}+(M / N)^{1 / 2}\right) \\
& \ll e^{-C(\log M)^{\delta}}+e^{C(\log M)^{7 \delta}}\left(M^{-1 / 2}+(M / N)^{1 / 2}\right) .
\end{aligned}
$$

Finally we estimate $I_{2}$ and $I_{3}$. Clearly,

$$
\begin{aligned}
\left|U_{L}(t, x, I)\right| & \leq\left|\exp \left(i t S_{I} / \sigma_{I}\right)\right|+\frac{|t|^{L}}{L !}\left|S_{I} / \sigma_{I}\right|^{L} \\
& \leq 1+\frac{|t|^{L}}{L !}\left|S_{I} / \sigma_{I}\right|^{L}
\end{aligned}
$$

and thus

$$
\left|I_{2}\right| \leq \mathbb{E}\left(\frac{|s|^{L}}{L !}\left|S_{J} / \sigma_{J}\right|^{L}\right)+\mathbb{E}\left(\frac{|t|^{L}}{L !} \frac{|s|^{L}}{L !}\left|S_{I} / \sigma_{I}\right|^{L}\left|S_{J} / \sigma_{J}\right|^{L}\right) .
$$

Here the second summand can be estimated in exactly the same way as $I_{4}$ and the first one can be estimated by using Lemma 2, Thus we get

$$
\left|I_{2}\right| \ll e^{-C(\log M)^{\delta}}+e^{C(\log M)^{7 \delta}}\left(M^{-1 / 2}+(M / N)^{1 / 2}\right) .
$$

A similar bound holds for $I_{3}$, and this completes the proof of Lemma 4

Lemma 5. Let $F$ and $G$ be probability distributions on $\mathbb{R}^{2}$ with characteristic functions $\varphi$ and $\gamma$, respectively, and let $T>0$. Then there exists a probability distribution $H$ on $\mathbb{R}^{2}$ such that $H\left(|x| \geq T^{-1 / 2} \log T\right) \ll e^{-T / 2}$, and for any Borel set $B \subset[-T, T]^{2}$,

$$
|(F * H)(B)-(G * H)(B)| \ll T^{2} \int_{[-T, T]^{2}}|\varphi(u)-\gamma(u)| d u+e^{-(\log T)^{2} / 4} .
$$

The constants implied by $\ll$ are absolute.

Proof. Let $\zeta_{0}$ be a standard $N(0, \mathbf{I})$ random variable in $\mathbb{R}^{2}$ and $\zeta=\frac{\log T}{T} \zeta_{0}$. Clearly, we have

$$
P\left(|\zeta| \geq \frac{\log T}{\sqrt{T}}\right)=P\left(\left|\zeta_{0}\right| \geq \sqrt{T}\right) \ll e^{-T / 2} .
$$


Letting $\psi$ and $H$ denote, respectively, the characteristic function and distribution of $\zeta$, we get

$$
\begin{aligned}
\left|f_{F * H}(x)-f_{G * H}(x)\right| & \leq(2 \pi)^{-1} \int_{\mathbb{R}^{2}}|\varphi(u)-\gamma(u)||\psi(u)| d u \\
& \leq \int_{[-T, T]^{2}}|\varphi(u)-\gamma(u)| d u+2 \int_{u \notin[-T, T]^{2}}|\psi(u)| d u,
\end{aligned}
$$

where $f_{F * H}$ and $f_{G * H}$ denote the density functions corresponding to the distributions $F * H$ and $G * H$, respectively. Letting $\tau=T^{-1} \log T$, we clearly have $\psi(u)=e^{-\tau^{2}|u|^{2} / 2}$ for $u \in \mathbb{R}^{2}$, and a simple calculation shows

$$
\int_{u \notin[-T, T]}|\psi(u)| d u \ll e^{-(\log T)^{2} / 3} .
$$

Thus

$$
\left|f_{F * H}(x)-f_{G * H}(x)\right| \ll \int_{[-T, T]^{2}}|\varphi(u)-\gamma(u)| d u+e^{-(\log T)^{2} / 3} \quad \text { for all } x \in \mathbb{R}^{2},
$$

whence for $B \subseteq[-T, T]^{2}$ we get

$$
|(F * H)(B)-(G * H)(B)| \ll T^{2} \int_{[-T, T]}|\varphi(u)-\gamma(u)| d u+T^{2} e^{-(\log T)^{2} / 3},
$$

proving Lemma 5

Lemma 6. Under the conditions of Lemma 3 we have for any $0<\delta<1$ and for $|x|,|y| \leq \frac{1}{8}(\log M)^{\delta / 2}$,

$$
\begin{aligned}
& \left|P\left(S_{I} / \sigma_{I} \leq x, S_{J} / \sigma_{J} \leq y\right)-\Phi(\hat{x}) \Phi(\hat{y})\right| \\
& \ll e^{-C(\log \log M)^{2}}+e^{C(\log M)^{7 \delta}}\left(M^{-1 / 2}+(M / N)^{1 / 2}\right),
\end{aligned}
$$

where $\Phi$ is the standard normal distribution function and $\hat{x}, \hat{y}$ are suitable numbers with $|\hat{x}-x| \leq C(\log M)^{-\delta / 8}$ and $|\hat{y}-y| \leq C(\log M)^{-\delta / 8}$.

Proof. Let

$$
F=\operatorname{dist}\left(S_{I} / \sigma_{I}, S_{J} / \sigma_{J}\right), \quad G=N(0, \mathbf{I}), \quad T=(\log M)^{\delta / 2} .
$$

By Lemmas 4 and 5 we have for any Borel set $B \subseteq[-T, T]^{2}$,

$$
\begin{aligned}
& |(F * H)(B)-(G * H)(B)| \\
& \ll T^{2} \int_{[-T, T]^{2}}|\varphi(u)-\gamma(u)| d u+e^{-(\log T)^{2} / 4} \\
& \ll(\log M)^{2 \delta}\left[e^{-C(\log M)^{\delta}}+e^{C(\log M)^{7 \delta}}\left(M^{-1 / 2}+(M / N)^{1 / 2}\right)\right]+e^{-c(\log \log M)^{2}} \\
& \ll e^{-C(\log M)^{\delta}}+e^{C(\log M)^{7 \delta}}\left(M^{-1 / 2}+(M / N)^{1 / 2}\right)+e^{-C(\log \log M)^{2}},
\end{aligned}
$$

where $H$ is a distribution on $\mathbb{R}^{2}$ such that

$$
H\left(x:|x| \geq C(\log M)^{-\delta / 8}\right) \leq e^{-C(\log M)^{\delta / 2}} .
$$


Applying Lemma 2 with $p=2[\log \log M]$ and using the Markov inequality, we get

$$
\begin{aligned}
& P\left(\left|S_{I} / \sigma_{I}\right| \geq T\right) \leq T^{-p} \mathbb{E}\left(\left|S_{I} / \sigma_{I}\right|^{p}\right) \ll T^{-p} \frac{p !}{(p / 2) !} 2^{-p / 2} \\
& \ll 4^{p}(\log M)^{-\delta p / 2} p^{p}=4^{p} \exp \left(p \log p-\frac{\delta p}{2} \log \log M\right) \\
& \ll \exp \left(-C(\log \log M)^{2}\right),
\end{aligned}
$$

and a similar inequality holds for $P\left(\left|S_{J} / \sigma_{J}\right| \geq T\right)$. Convolution with $H$ means adding an (independent) r.v. which is $<C(\log M)^{-\delta / 8}$ with the exception of a set with probability $e^{-C(\log M)^{\delta / 2}}$. Thus choosing $B=[-T, x] \times[-T, y]$ with $|x| \leq$ $C(\log M)^{\delta / 2}$ and $|y| \leq C(\log M)^{\delta / 2}$ we get

$$
\begin{aligned}
& \left|P\left(S_{I} / \sigma_{I} \leq x, S_{J} / \sigma_{J} \leq y\right)-\Phi(\hat{x}) \Phi(\hat{y})\right| \\
& \ll e^{-C(\log \log M)^{2}}+e^{C(\log M)^{7 \delta}}\left(M^{-1 / 2}+(M / N)^{1 / 2}\right),
\end{aligned}
$$

where $|\hat{x}-x| \leq C(\log M)^{-\delta / 8}$ and $|\hat{y}-y| \leq C(\log M)^{-\delta / 8}$.

Remark. The one-dimensional analogue of Lemma 6 can be proved in the same way (in fact, the argument is much simpler):

$$
\left|P\left(S_{I} / \sigma_{I} \leq x\right)-\Phi(\hat{x})\right| \ll e^{-C(\log \log M)^{2}}
$$

for $|x| \leq \frac{1}{8}(\log M)^{\delta / 2}$, where $|\hat{x}-x| \leq C(\log M)^{-\delta / 8}$. Using this fact, the statement of Lemma 6 and simple algebra show that for $|x|,|y| \leq \frac{1}{8}(\log M)^{\delta / 2}$ we have

$$
\begin{aligned}
& \left|P\left(S_{I} / \sigma_{I}>x, S_{J} / \sigma_{J}>y\right)-\Psi(\hat{x}) \Psi(\hat{y})\right| \\
& \ll e^{-C(\log \log M)^{2}}+e^{C(\log M)^{7 \delta}}\left(M^{-1 / 2}+(M / N)^{1 / 2}\right),
\end{aligned}
$$

where $\hat{x}$ and $\hat{y}$ are suitable numbers with $|\hat{x}-x| \leq C(\log M)^{-\delta / 8}$ and $|\hat{y}-y| \leq$ $C(\log M)^{-\delta / 8}$. Here $\Psi(x)=1-\Phi(x)$.

\section{Proof of Theorem 1}

The CLT (1.5) in Theorem 1 follows immediately from Lemma 6. see also the Remark after Lemma 6. To prove the LIL (1.6), assume the conditions of Theorem 1 and let $\sigma: \mathbb{N} \rightarrow \mathbb{N}$ be a permutation of $\mathbb{N}$. Clearly, for $p=O(\log \log N)$ we have $\exp \left(C p^{7}\right) \ll N^{1 / 4}$, and thus Lemma 2 implies

$$
\int_{0}^{1}\left(\sum_{k=M+1}^{M+N} f\left(n_{\sigma(k)} x\right)\right)^{2 p} d x \sim \frac{(2 p) !}{p !} 2^{-p}\left(1+O\left(N^{-1 / 4}\right)\right) A_{N, M}^{p} \quad \text { as } N \rightarrow \infty
$$

uniformly for $p=O(\log \log N)$ and $M \geq 1$. Using this fact, the upper half of the LIL (1.6) can be proved by following the classical proof of Erdös and Gál [4] of the LIL for lacunary trigonometric series. (The observation that the upper half of the LIL follows from asymptotic moment estimates was already used by Philipp [17] to prove the LIL for mixing sequences.) To prove the lower half of the LIL we first observe that the upper half of the LIL and relation (2.2) imply

$$
\limsup _{N \rightarrow \infty}(N \log \log N)^{-1 / 2} \sum_{k=1}^{N} f\left(n_{\sigma(k)} x\right) \leq K\|f\|^{1 / 8} \quad \text { a.e., }
$$


where $K$ is a constant depending on the generating elements of $\left(n_{k}\right)$. Given any $f$ satisfying (1.3) and $\varepsilon>0, f$ can be written as $f=f_{1}+f_{2}$, where $f_{1}$ is a trigonometric polynomial and $\left\|f_{2}\right\| \leq \varepsilon$, and thus applying (3.1) with $f=f_{2}$ it is immediately seen that it suffices to prove the lower half of the LIL for trigonometric polynomials $f$.

Let $\theta \geq 2$ be an integer and set

$$
\eta_{n}=\frac{X_{\theta^{n}+1}+\cdots+X_{\theta^{n+1}}}{\gamma_{n}}
$$

where $X_{j}=f\left(n_{\sigma(j)} x\right)$ and $\gamma_{n}^{2}=\operatorname{Var}\left(X_{\theta^{n}+1}+\cdots+X_{\theta^{n+1}}\right)$. Fix $\varepsilon>0$ and put

$$
A_{n}=\left\{\eta_{n} \geq(1-\varepsilon)\left(2 \log \log \gamma_{n}\right)^{1 / 2}\right\} \text {. }
$$

We will prove that $P\left(A_{n}\right.$ i.o. $)=1$. Here we use an idea of Révész 19 and the following generalization of the Borel-Cantelli lemma; see Spitzer [21], p. 317.

Lemma 7. Let $A_{n}, n=1,2, \ldots$, be events satisfying $\sum_{n=1}^{\infty} P\left(A_{n}\right)=\infty$ and

$$
\lim _{N \rightarrow \infty} \frac{\sum_{1 \leq m, n \leq N}\left|P\left(A_{m} \cap A_{n}\right)-P\left(A_{m}\right) P\left(A_{n}\right)\right|}{\left(\sum_{n=1}^{N} P\left(A_{n}\right)\right)^{2}}=0 .
$$

Then $P\left(A_{n}\right.$ i.o. $)=1$.

By the one-dimensional version of Lemma 6 (see the Remark at the end of Section 2) we have

$$
P\left(A_{n}\right)=\Psi\left((1-\varepsilon)\left(2 \log \log \gamma_{n}\right)^{\frac{1}{2}}+z_{n}\right)+O\left(e^{-C(\log n)^{2}}\right),
$$

where $\left|z_{n}\right| \leq C n^{-\delta / 8}$. By the mean value theorem, $\Psi(x) \sim(2 \pi)^{-1 / 2} x^{-1} \exp \left(-x^{2} / 2\right)$ and $\theta^{n} \ll \gamma_{n}^{2} \ll \theta^{n}$ we have

$$
\begin{aligned}
\Psi & \left((1-\varepsilon)\left(2 \log \log \gamma_{n}\right)^{1 / 2}+z_{n}\right) \\
= & \Psi\left((1-\varepsilon)\left(2 \log \log \gamma_{n}\right)^{1 / 2}\right) \\
& +\exp \left(-\frac{1}{2}\left[(1-\varepsilon)\left(2 \log \log \gamma_{n}\right)^{1 / 2}+O(1) n^{-\delta / 8}\right]^{2}\right) O\left(n^{-\delta / 8}\right) \\
= & \Psi\left((1-\varepsilon)\left(2 \log \log \gamma_{n}\right)^{1 / 2}\right) \\
& +\exp \left(-(1-\varepsilon)^{2} \log \log \gamma_{n}+O(1)\right) O\left(n^{-\delta / 8}\right) \\
= & \Psi\left((1-\varepsilon)\left(2 \log \log \gamma_{n}\right)^{1 / 2}\right) \\
& +O(1) \Psi\left((1-\varepsilon)\left(2 \log \log \gamma_{n}\right)^{1 / 2}\right)(\log n)^{1 / 2} n^{-\delta / 8}
\end{aligned}
$$

In particular,

$$
\Psi\left((1-\varepsilon)\left(2 \log \log \gamma_{n}\right)^{1 / 2}+z_{n}\right) \sim \Psi\left((1-\varepsilon)\left(2 \log \log \gamma_{n}\right)^{1 / 2}\right),
$$

and thus (3.2) implies

$$
P\left(A_{n}\right) \sim \Psi\left((1-\varepsilon)\left(2 \log \log \gamma_{n}\right)^{1 / 2}\right) \gg \frac{1}{n^{(1-\varepsilon)^{2}}(\log n)^{1 / 2}} .
$$

Hence the estimates in (3.3) yield

$$
\begin{aligned}
& \Psi\left((1-\varepsilon)\left(2 \log \log \gamma_{n}\right)^{1 / 2}+z_{n}\right) \\
& \quad=\Psi\left((1-\varepsilon)\left(2 \log \log \gamma_{n}\right)^{1 / 2}\right)+O\left(P\left(A_{n}\right) n^{-\delta / 16}\right) .
\end{aligned}
$$


Now by Lemma 6 for $m \leq n$ (see the Remark at the end of Section 2)

$$
\begin{aligned}
P & \left(A_{m} \cap A_{n}\right) \\
& =\Psi\left((1-\varepsilon)\left(2 \log \log \gamma_{m}\right)^{1 / 2}+z_{1}\right) \Psi\left((1-\varepsilon)\left(2 \log \log \gamma_{n}\right)^{1 / 2}+z_{2}\right) \\
& +O(1)\left[e^{-C(\log m)^{2}}+e^{C m^{7 \delta}}\left(e^{-C m}+e^{-C(n-m)}\right)\right],
\end{aligned}
$$

provided $\log n \leq m^{\delta / 2}$. The expression $\Psi(\ldots) \Psi(\ldots)$ in (3.6) equals by (3.4) and (3.5),

$\Psi\left((1-\varepsilon)\left(2 \log \log \gamma_{m}\right)^{1 / 2}\right) \Psi\left((1-\varepsilon)\left(2 \log \log \gamma_{n}\right)^{1 / 2}\right)+O\left(P\left(A_{m}\right) P\left(A_{n}\right) m^{-\delta / 16}\right)$.

Hence, also assuming $n-m \geq m^{8 \delta}$ we get from (3.6),

$$
\begin{aligned}
P\left(A_{m} \cap A_{n}\right)= & \Psi\left((1-\varepsilon)\left(2 \log \log \gamma_{m}\right)^{1 / 2}\right) \Psi\left((1-\varepsilon)\left(2 \log \log \gamma_{n}\right)^{1 / 2}\right) \\
& +O\left(P\left(A_{m}\right) P\left(A_{n}\right) m^{-\delta / 16}\right)+O\left(e^{-C(\log m)^{2}}\right) .
\end{aligned}
$$

Further, by (3.2) and the above estimates

$$
\begin{aligned}
P\left(A_{m}\right) P\left(A_{n}\right)= & \Psi\left((1-\varepsilon)\left(2 \log \log \gamma_{m}\right)^{1 / 2}\right) \Psi\left((1-\varepsilon)\left(2 \log \log \gamma_{n}\right)^{1 / 2}\right) \\
& +O\left(P\left(A_{m}\right) P\left(A_{n}\right) m^{-\delta / 16}\right)+O\left(e^{-C(\log m)^{2}}\right),
\end{aligned}
$$

and thus we obtained the following lemma.

Lemma 8. We have

$$
\left|P\left(A_{m} \cap A_{n}\right)-P\left(A_{m}\right) P\left(A_{n}\right)\right| \ll P\left(A_{m}\right) P\left(A_{n}\right) m^{-\delta / 16}+e^{-C(\log m)^{2}},
$$

provided $n-m \geq m^{8 \delta}$ and $\log n \leq m^{\delta}$.

We can now prove the following lemma.

Lemma 9. We have

$$
\lim _{N \rightarrow \infty} \frac{\sum_{1 \leq m, n \leq N}\left|P\left(A_{m} \cap A_{n}\right)-P\left(A_{m}\right) P\left(A_{n}\right)\right|}{\left(\sum_{n=1}^{N} P\left(A_{n}\right)\right)^{2}}=0 .
$$

Proof. By Lemma 8 we have

$$
\begin{aligned}
& \sum_{\substack{1 \leq m, n \leq N \\
n-m \geq m^{8 \delta} \\
m \geq C N^{\delta}}}\left|P\left(A_{m} \cap A_{n}\right)-P\left(A_{m}\right) P\left(A_{n}\right)\right| \\
\ll & \left(\sum_{m=1}^{N} P\left(A_{m}\right) m^{-\delta / 16}\right)\left(\sum_{n=1}^{N} P\left(A_{n}\right)\right)+N^{2} e^{-c(\log N)^{2}} \\
= & o_{N}(1)\left(\sum_{m=1}^{N} P\left(A_{m}\right)\right)\left(\sum_{n=1}^{N} P\left(A_{n}\right)\right)+O(1)=o_{N}(1)\left(\sum_{m=1}^{N} P\left(A_{m}\right)\right)^{2},
\end{aligned}
$$


since $\sum_{n=1}^{N} P\left(A_{n}\right)=+\infty$ by (3.4). Further,

$$
\begin{aligned}
& \sum_{\substack{1 \leq m, n \leq N \\
0 \leq n-m \leq m^{8 \delta}}}\left|P\left(A_{m} \cap A_{n}\right)-P\left(A_{m}\right) P\left(A_{n}\right)\right| \\
\leq & \sum_{\substack{1 \leq m, n \leq N \\
m \leq n \leq m+m^{8 \delta}}} 2 P\left(A_{m}\right) \leq 2 \sum_{m=1}^{N} m^{8 \delta} P\left(A_{m}\right) \leq 2 N^{8 \delta} \sum_{m=1}^{N} P\left(A_{m}\right)
\end{aligned}
$$

and

$$
\sum_{\substack{1 \leq m \leq n \leq N \\ m \leq C N^{\delta}}}\left|P\left(A_{m} \cap A_{n}\right)-P\left(A_{m}\right) P\left(A_{n}\right)\right| \leq \sum_{\substack{1 \leq m \leq n \leq N \\ m \leq C N^{\delta}}} 2 P\left(A_{n}\right) \leq 2 N^{\delta} \sum_{n=1}^{N} P\left(A_{n}\right) .
$$

The previous estimates imply

$$
\begin{aligned}
& \sum_{1 \leq m, n \leq N}\left|P\left(A_{m} \cap A_{n}\right)-P\left(A_{m}\right) P\left(A_{n}\right)\right| \\
& \ll o_{N}(1)\left(\sum_{n=1}^{N} P\left(A_{n}\right)\right)^{2}+N^{8 \delta}\left(\sum_{n=1}^{N} P\left(A_{n}\right)\right) .
\end{aligned}
$$

Since

$$
\sum_{n=1}^{N} P\left(A_{n}\right) \gg \sum_{n=1}^{N} \frac{1}{n^{(1-\varepsilon)^{2}}(\log n)^{1 / 2}} \gg \sum_{n=1}^{N} \frac{1}{n^{1-\varepsilon}} \gg N^{\varepsilon},
$$

choosing $\delta<\varepsilon / 8$ we get

$$
\frac{\sum_{1 \leq m, n \leq N}\left|P\left(A_{m} \cap A_{n}\right)-P\left(A_{m}\right) P\left(A_{n}\right)\right|}{\left(\sum_{n=1}^{N} P\left(A_{n}\right)\right)^{2}} \ll o_{N}(1)+\frac{N^{8 \delta}}{\sum_{n=1}^{N} P\left(A_{n}\right)} \rightarrow 0 .
$$

We can now complete the proof of the lower half of the LIL. By Lemmas 7 and 9 and we have with probability 1

$$
\left|X_{\theta^{n}+1}+\cdots+X_{\theta^{n+1}}\right| \geq(1-\varepsilon)\left(2 \gamma_{n}^{2} \log \log \gamma_{n}\right)^{1 / 2} \quad \text { i.o., }
$$

where $\gamma_{n}=\left\|X_{\theta^{n}+1}+\cdots+X_{\theta^{n+1}}\right\|$. By the already proved upper half of the LIL we have

$$
\left|X_{1}+\cdots+X_{\theta^{n}}\right| \leq(1+\varepsilon)\left(2 A_{\theta^{n}}^{2} \log \log A_{\theta^{n}}\right)^{1 / 2} \quad \text { a.s. }
$$

and (2.2) and the assumptions of Theorem 1 imply

$$
A_{\theta^{n+1}} / A_{\theta^{n}} \geq C \theta^{1 / 2},
$$


whence

$$
\begin{aligned}
\gamma_{n} & \geq\left\|X_{1}+\cdots+X_{\theta^{n+1}}\right\|-\left\|X_{1}+\cdots+X_{\theta^{n}}\right\|=A_{\theta^{n+1}}-A_{\theta^{n}} \\
& \geq A_{\theta^{n+1}}\left(1-O\left(\theta^{-1 / 2}\right)\right) .
\end{aligned}
$$

Thus using (3.8), (3.9), (3.10) and (3.11) we get with probability 1 for infinitely many $n$

$$
\begin{aligned}
& \left|X_{1}+\cdots+X_{\theta^{n+1}}\right| \\
& \geq(1-\varepsilon)\left(2 \gamma_{n}^{2} \log \log \gamma_{n}\right)^{1 / 2}-(1+\varepsilon)\left(2 A_{\theta^{n}}^{2} \log \log A_{\theta^{n}}\right)^{1 / 2} \\
& \geq(1-2 \varepsilon)\left(2 A_{\theta^{n+1}}^{2} \log \log A_{\theta^{n+1}}\right)^{1 / 2},
\end{aligned}
$$

provided we choose $\theta=\theta(\varepsilon)$ large enough. This completes the proof of the lower half of the LIL.

To prove the Corollary, assume that

$$
N^{-1 / 2} \sum_{k=1}^{N} f\left(n_{\sigma(k)} x\right) \stackrel{d}{\longrightarrow} G
$$

with a nondegenerate distribution $G$. By Lemma 2 and (2.2) we have

$$
\mathbb{E}\left(\sum_{k=1}^{N} f\left(n_{\sigma(k)} x\right)\right)^{4} \ll N^{2}
$$

and thus the sequence

$$
N^{-1}\left(\sum_{k=1}^{N} f\left(n_{\sigma(k)} x\right)\right)^{2}, \quad N=1,2, \ldots,
$$

is bounded in $L_{2}$ norm and consequently uniformly integrable. Thus the second moment of the left hand side of (3.12) converges to the second moment $\gamma^{2}$ of $G$, which is nonzero, since $G$ is nondegenerate. Thus we proved (1.8), and since the nonnegativity of the Fourier coefficients of $f$ implies (1.4), Theorem 1 yields (1.9) and (1.10).

We now prove a remark made at the end of the Introduction concerning the set $\Gamma_{f}$ of limiting variances corresponding to all permutations $\sigma$. Let $f$ be a function satisfying (1.3) with nonnegative Fourier coefficients. Assume that $f$ is even, i.e. its Fourier series

$$
f(x) \sim \sum_{j=1}^{\infty} a_{j} \cos 2 \pi j x
$$

is a pure cosine series; the general case requires only trivial changes. Note that the Fourier coefficients of $f$ satisfy (2.3), and by Kac [12] we have

$$
\int_{0}^{1}\left(\sum_{k=1}^{N} f\left(2^{k} x\right)\right)^{2} d x \sim \gamma_{f}^{2} N
$$

where

$$
\gamma_{f}^{2}=\|f\|^{2}+2 \sum_{r=1}^{\infty} \int_{0}^{1} f(x) f\left(2^{r} x\right) d x \geq\|f\|^{2}
$$


We first note that for any permutation $\sigma: \mathbb{N} \rightarrow \mathbb{N}$ we have

$$
\|f\|^{2} \leq \frac{1}{N} \int_{0}^{1}\left(\sum_{k=1}^{N} f\left(2^{\sigma(k)} x\right)\right)^{2} d x \leq \gamma_{f}^{2}
$$

for any $N \geq 1$. To see this, we observe that

$$
\begin{aligned}
& \int_{0}^{1}\left(\sum_{k=1}^{N} f\left(2^{\sigma(k)} x\right)\right)^{2} d x \\
& =N\|f\|^{2}+\sum_{1 \leq i \neq j \leq N} \int_{0}^{1} f\left(2^{\sigma(i)} x\right) f\left(2^{\sigma(j)} x\right) d x \\
& =N\|f\|^{2}+\sum_{1 \leq i \neq j \leq N} \int_{0}^{1} f(x) f\left(2^{|\sigma(j)-\sigma(i)|} x\right) d x \\
& =N\|f\|^{2}+\sum_{r=1}^{\infty} a_{r}^{(N)} \int_{0}^{1} f(x) f\left(2^{r} x\right) d x,
\end{aligned}
$$

where

$$
a_{r}^{(N)}=\#\{1 \leq i \neq j \leq N:|\sigma(j)-\sigma(i)|=r\}
$$

Fix $r \geq 1$. Clearly, for any $1 \leq i \leq N$ there exist at most two indices $1 \leq j \leq N$, $j \neq i$, such that $|\sigma(j)-\sigma(i)|=r$. Hence $a_{r}^{(N)} \leq 2 N$, and by the nonnegativity of the Fourier coefficients of $f$, the integrals in the last line of (3.14) are nonnegative. Thus (3.13) is proved. Next we claim that for any $\rho \in\left[\|f\|, \gamma_{f}\right]$ we can find a permutation $\sigma: \mathbb{N} \rightarrow \mathbb{N}$ such that

$$
\int_{0}^{1}\left(\sum_{k=1}^{N} f\left(2^{\sigma(k)} x\right)\right)^{2} d x \sim \rho^{2} N
$$

To this end, we will need the following lemma.

Lemma 10. For some $J \geq 0$ let

$$
g(x)=\sum_{j=J+1}^{\infty} a_{j} \cos 2 \pi j x
$$

Then for any set $\left\{m_{1}, \ldots, m_{N}\right\}$ of distinct positive integers we have

$$
\left\|\sum_{k=1}^{N} g\left(2^{m_{k}} x\right)\right\| \leq \begin{cases}2 \sqrt{N} & \text { for } J=0 \\ \sqrt{2 N} J^{-1 / 2} & \text { for } J \geq 1\end{cases}
$$


Proof. Similar to (2.5) we have

$$
\begin{aligned}
& \int_{0}^{1}\left(\sum_{k=1}^{N} g\left(2^{m_{k}} x\right)\right)^{2} d x \\
= & \frac{1}{2} \sum_{1 \leq k_{1}, k_{2} \leq N} \sum_{j_{1}, j_{2} \geq J+1} a_{j_{1}} a_{j_{2}} \cdot \mathbf{1}\left(j_{1} 2^{m_{k_{1}}}=j_{2} 2^{m_{k_{2}}}\right) \\
\leq & \sum_{1 \leq k_{1} \leq k_{2} \leq N} \sum_{j_{1}, j_{2} \geq J+1} \frac{1}{j_{1} j_{2}} \cdot \mathbf{1}\left(j_{1} 2^{m_{k_{1}}}=j_{2} 2^{m_{k_{2}}}\right) \\
\leq & \sum_{1 \leq k_{1} \leq k_{2} \leq N} \sum_{j \geq J+1} \frac{2^{m_{k_{1}}}}{j^{2} 2^{m_{k_{2}}}} \\
\leq & N \sum_{v=0}^{\infty} 2^{-v} \sum_{j \geq J+1} \frac{1}{j^{2}} \\
\leq & \begin{cases}4 N & \text { for } J=0, \\
2 N J^{-1} & \text { for } J \geq 1 .\end{cases}
\end{aligned}
$$

Now let $\rho \in\left[\|f\|, \gamma_{f}\right]$ be given and write

$$
\alpha=\frac{\rho^{2}-\|f\|^{2}}{\gamma_{f}^{2}-\|f\|^{2}} .
$$

Clearly,

$$
\alpha \in[0,1] .
$$

Postponing the extremal cases $\alpha=0$ and $\alpha=1$, assume $\alpha \in(0,1)$. Set

$$
\Delta_{i}=\left\{i^{2}+1, \ldots,(i+1)^{2}\right\}, \quad i \geq 0 .
$$

For every positive integer $k$ there exists exactly one number $i=i(k)$ such that $k \in \Delta_{i}$. Now we set $n_{1}=1$ and define a sequence $\left(n_{k}\right)_{k \geq 1}$ recursively by

$$
n_{k}=\left\{\begin{array}{lll}
n_{k-1}+i+1 & \text { if } & k=i^{2}+1 \text { for some } i \\
n_{k-1}+1 & \text { if } & k \in\left\{i^{2}+2, i^{2}+\lceil 2 i \alpha\rceil\right\} \text { for some } i \\
n_{k-1}+i+1 & & \text { otherwise }
\end{array}\right.
$$

For any $i \geq 0$, set

$$
p^{(i)}(x)=\sum_{j=1}^{2^{i}} a_{j} \cos 2 \pi j x, \quad r^{(i)}(x)=f(x)-p^{(i)}(x)=\sum_{j=2^{i}+1}^{\infty} a_{j} \cos 2 \pi j x .
$$

We want to calculate

$$
\int_{0}^{1}\left(\sum_{k=1}^{N} f\left(2^{n_{k}} x\right)\right)^{2} d x
$$

asymptotically. There is an $i$ such that $N \in \Delta_{i}$, and since $N-i^{2} \leq(i+1)^{2}-i^{2}=$ $2 i+1 \leq 2 \sqrt{N}+1$, we have by Lemma 10

$$
\left\|\sum_{k=1}^{N} f\left(2^{n_{k}} x\right)\right\|-\left\|\sum_{k=1}^{i^{2}} f\left(2^{n_{k}} x\right)\right\| \leq\left\|\sum_{k=i^{2}+1}^{N} f\left(2^{n_{k}} x\right)\right\| \leq 2(2 \sqrt{N}+1)^{1 / 2}
$$


Using Lemma 10 again, we get

$$
\begin{aligned}
& \left\|\sum_{k=1}^{i^{2}} f\left(2^{n_{k}} x\right)\right\| \\
= & \left\|\sum_{h=0}^{i-1}\left(\sum_{k \in \Delta_{h}} p^{(h)}\left(2^{n_{k}} x\right)+\sum_{k \in \Delta_{h}} r^{(h)}\left(2^{n_{k}} x\right)\right)\right\|
\end{aligned}
$$

and

$$
\begin{aligned}
& \left\|\sum_{k=1}^{i^{2}} f\left(2^{n_{k}} x\right)\right\|-\left\|\sum_{h=0}^{i-1}\left(\sum_{k \in \Delta_{h}} p^{(h)}\left(2^{n_{k}} x\right)\right)\right\| \\
\leq & \sum_{h=0}^{i-1}\left\|\left(\sum_{k \in \Delta_{h}} r^{(h)}\left(2^{n_{k}} x\right)\right)\right\| \\
\leq & \sum_{h=0}^{i-1} \sqrt{2\left|\Delta_{h}\right|} 2^{-h / 2} \\
\leq & \sum_{h=0}^{i-1} \sqrt{2(2 h+1)} 2^{-h / 2} \\
\ll & 1 .
\end{aligned}
$$

Now we calculate

$$
\left\|\sum_{h=0}^{i-1}\left(\sum_{k \in \Delta_{h}} p^{(h)}\left(2^{n_{k}} x\right)\right)\right\| .
$$

By the construction of the sequence $\left(n_{k}\right)_{k \geq 1}$, the functions

$$
\sum_{k \in \Delta_{h_{1}}} p^{\left(h_{1}\right)}\left(2^{n_{k}} x\right), \quad \sum_{k \in \Delta_{h_{2}}} p^{\left(h_{2}\right)}\left(2^{n_{k}} x\right)
$$

are orthogonal if $h_{1} \neq h_{2}$. In fact, if $h_{2}>h_{1}$, and $k_{1} \in \Delta_{h_{1}}, k_{2} \in \Delta_{h_{2}}$, then $n_{k_{2}} \geq n_{k_{1}}+h_{2}+1$, which implies that the largest frequency of a trigonometric function in the Fourier series of $p^{\left(h_{1}\right)}\left(2^{n_{k_{1}}} x\right)$ is $2^{h_{1}} 2^{n_{k_{1}}}<2^{n_{k_{2}}}$. Thus the functions in (3.18) are really orthogonal. A similar argument shows that for fixed $h$ and $k_{1}, k_{2} \in \Delta_{h}$ the functions $p^{(h)}\left(2^{n_{k_{1}}} x\right)$ and $p^{(h)}\left(2^{n_{k_{2}}} x\right)$ are orthogonal if both $k_{1}$ and $k_{2}$ are not in the set $\left\{h^{2}+1, h^{2}+\lceil 2 h \alpha\rceil\right\}$. Thus

$$
\begin{aligned}
& \int_{0}^{1}\left(\sum_{h=0}^{i-1}\left(\sum_{k \in \Delta_{h}} p^{(h)}\left(2^{n_{k}} x\right)\right)\right)^{2} \\
&=\sum_{h=0}^{i-1}\left(\int_{0}^{1}\left(\sum_{k \in\left\{h^{2}+1, h^{2}+\lceil 2 h \alpha\rceil\right\}} p^{(h)}\left(2^{n_{k}} x\right)\right)^{2} d x\right. \\
&\left.+\sum_{k \in\left\{h^{2}+\lceil 2 h \alpha\rceil+1,(h+1)^{2}\right\}}\left\|p^{(h)}\right\|^{2}\right) .
\end{aligned}
$$


For $h \rightarrow \infty$,

$$
\int_{0}^{1}\left(\sum_{k \in\left\{h^{2}+1, h^{2}+\lceil 2 h \alpha\rceil\right\}} p^{(h)}\left(2^{n_{k}} x\right)\right)^{2} d x \sim \gamma_{f}^{2}\left(h^{2}+\lceil 2 h \alpha\rceil-\left(h^{2}+1\right)\right) \sim \gamma_{f}^{2} 2 h \alpha
$$

and

$$
\sum_{k \in\left\{h^{2}+\lceil 2 h \alpha\rceil+1,(h+1)^{2}\right\}}\left\|p^{(h)}\right\|^{2} \sim\|f\|^{2}\left((h+1)^{2}-\left(h^{2}+\lceil 2 h \alpha\rceil\right)\right) \sim\|f\|^{2} 2 h(1-\alpha) .
$$

Thus by (3.19) for $i \rightarrow \infty$

$$
\begin{aligned}
& \int_{0}^{1}\left(\sum_{h=0}^{i-1}\left(\sum_{k \in \Delta_{h}} p^{(h)}\left(2^{n_{k}} x\right)\right)\right)^{2} \\
\sim & \sum_{h=0}^{i-1}\left(\gamma_{f}^{2} 2 h \alpha+\|f\|^{2} 2 h(1-\alpha)\right) \\
\sim & \left(\gamma_{f}^{2} \alpha+\|f\|^{2}(1-\alpha)\right) \sum_{h=0}^{i-1} 2 h \\
\sim & \rho^{2} i^{2} .
\end{aligned}
$$

Combining (3.16), (3.17) and (3.20) we finally obtain

$$
\int_{0}^{1}\left(\sum_{k=1}^{N} f\left(2^{n_{k}} x\right)\right)^{2} d x \sim \rho^{2} N \quad \text { as } N \rightarrow \infty .
$$

Note that in our argument we assumed $\alpha \in(0,1)$, i.e. that $\rho$ is an inner point of the interval $\left[\|f\|, \gamma_{f}\right]$. The case $\alpha=1$ (i.e. $\rho=\gamma_{f}$ ) is trivial, with $n_{k}=k$. In the case $\alpha=0$ we choose $\left(n_{k}\right)$ growing very rapidly, and the theory of lacunary series implies (3.21) with $\rho=\|f\|$.

Relation (3.21) is not identical with (3.15), since the sequence $\left(n_{k}\right)$ is not a permutation of $\mathbb{N}$. However, from $\left(n_{k}\right)$ we can easily construct a permutation $\sigma$ such that (3.15) holds. Let $H$ denote the set of positive integers not contained in $\left(n_{k}\right)$ and insert the elements of $H$ into the sequence $n_{1}, n_{2}, \ldots$ by leaving very rapidly increasing gaps between them. The so-obtained sequence is a permutation $\sigma$ of $\mathbb{N}$, and if the gaps between the inserted elements grow sufficiently rapidly, then clearly the asyimptotics of the integrals in (3.15) and (3.21) are the same, i.e. (3.15) holds. This completes the proof of the fact that the class of limits

$$
\lim _{N \rightarrow \infty} \frac{1}{N} \int_{0}^{1}\left(\sum_{k=1}^{N} f\left(2^{\sigma(k)} x\right)\right)^{2} d x
$$

is identical with the interval $\left[\|f\|^{2}, \gamma_{f}^{2}\right]$.

We next show that without assuming the nonnegativity of the Fourier coefficients of $f$, the class $\Gamma_{f}$ of limiting variances in Theorem 1 for permuted sequences 
$f\left(n_{\sigma(k)} x\right)$ is not necessarily the closed interval with endpoints $\|f\|^{2}$ and $\gamma_{f}^{2}$. Let

$$
f(x)=\cos 2 \pi x-\cos 4 \pi x+\cos 8 \pi x,
$$

and again $n_{k}=2^{k}$. Then taking into account the cancellations in the sum $\sum_{k=1}^{N} f\left(n_{k} x\right)$ we get

$$
\sum_{k=1}^{N} f\left(n_{k} x\right)=\cos 4 \pi x+\cos 16 \pi x+\cos 32 \pi x+\cos 64 \pi x+\cos 128 \pi x+\cdots,
$$

whence

$$
\int_{0}^{1}\left(\sum_{k=1}^{N} f\left(n_{k} x\right)\right)^{2} d x \sim N / 2
$$

so that $\gamma_{f}^{2}=1 / 2$ and clearly $\|f\|^{2}=3 / 2$. (Note that in this case $\gamma_{f}<\|f\|$.) Now

$$
\begin{aligned}
\sum_{k=1}^{N} f\left(4^{k} x\right) & =\cos 8 \pi x-\cos 16 \pi x \\
& +2 \cos 32 \pi x-\cos 64 \pi x+2 \cos 128 \pi x-\cos 256 x+2 \cos 512 x-\cdots
\end{aligned}
$$

and thus

$$
\int_{0}^{1}\left(\sum_{k=1}^{N} f\left(4^{k} x\right)\right)^{2} d x \sim 5 N / 2
$$

Similarly as above, we can get a permutation $\sigma$ of $\mathbb{N}$ such that

$$
\int_{0}^{1}\left(\sum_{k=1}^{N} f\left(2^{\sigma(k)} x\right)\right)^{2} d x \sim 5 N / 2
$$

In conclusion we prove the remark made at the end of the Introduction and construct a Hadamard lacunary sequence $\left(m_{k}\right)$ such that $f\left(m_{k} x\right)$ satisfies the CLT and LIL for all periodic Lipschitz functions $f$, but this property is destroyed by a suitable permutation of the sequence $f\left(m_{k} x\right)$. Let $\left(p_{k}\right)$ be a sequence of positive integers with $p_{k+1}-p_{k} \rightarrow \infty$ and let the sequence $\left(m_{k}\right)$ consist of the numbers $2^{p_{k}}-1, k=1,2, \ldots$, plus the numbers $2^{p_{k}+1}-1$ for the indices $k$ of the form $k=n^{3}$. Obviously, $\left(m_{k}\right)$ satisfies the Hadamard gap condition. Let $f$ be a periodic Lipschitz function with mean 0 and $\|f\|_{2}=1$. By results of Takahashi [23, 24], the CLT and LIL hold for $f\left(\left(2^{p_{k}}-1\right) x\right)$ with the standard norming sequences. Clearly $\sum_{k=1}^{N} f\left(m_{k} x\right)=\sum_{j=1}^{M} f\left(\left(2^{p_{j}}-1\right) x\right)+O\left(N^{1 / 3}\right)$, where $N-2 N^{1 / 3} \leq M \leq N$ for $N \geq N_{0}$, which implies that $f\left(m_{k} x\right)$ also satisfies the CLT and LIL. On the other hand, for infinitely many $\ell$ we have $m_{\ell}=2^{p_{k}}-1$ and $m_{\ell+1}=2^{p_{k}+1}-1$ for some $k$ and thus $m_{\ell+1}-2 m_{\ell}=1$. Thus $\left(m_{k}\right)$ contains a subsequence $\left(m_{k}^{*}\right)$ such that $m_{2 k}^{*}-2 m_{2 k-1}^{*}=1$ for $k=1,2, \ldots$ and $m_{2 k+1}^{*} / m_{2 k}^{*} \rightarrow \infty$ as $k \rightarrow \infty$. Thus using the method of Gaposhkin [11 it follows that setting $f(x)=\cos 2 \pi x+\cos 4 \pi x, N^{-1 / 2} \sum_{k=1}^{N} f\left(m_{k}^{*} x\right)$ has a nongaussian limit distribution, and a similar argument shows that $\lim \sup _{N \rightarrow \infty}(N \log \log N)^{-1 / 2} \sum_{k=1}^{N} f\left(n_{k} x\right)$ is a 
finite, nonconstant function a.e. In other words, $f\left(m_{k} x\right)$ has a subsequence failing both the CLT and LIL, and similarly as in the previous examples, this leads to a permutation of the sequence $f\left(m_{k} x\right)$ failing the CLT and LIL.

\section{REFERENCES}

[1] F. Amoroso and E. Viada, Small points on subvarieties of a torus. Duke Math. J. 150 (2009), 407-442. MR2582101 (2010m:11080)

[2] R.C. Baker, Riemann sums and Lebesgue integrals. Quart. J. Math. Oxford Ser. 258 (1976), 191-198. MR0409395 (53:13150)

[3] R.C. Bradley, A stationary rho-mixing Markov chain which is not "interlaced" rho-mixing. J. Theoret. Probab. 14 (2001), 717-727. MR.1860520 (2002k:60147)

[4] P. Erdős and I.S. Gál, On the law of the iterated logarithm. Proc. Nederl. Akad. Wetensch. Ser A 58 (1955), 65-84. MR0069309 (16:1016g)

[5] J.-H. Evertse, R. H.-P. Schlickewei and W. M. Schmidt, Linear equations in variables which lie in a multiplicative group, Ann. of Math. 155 (2002), 807-836. MR 1923966 (2003f:11037)

[6] K. Fukuyama, The law of the iterated logarithm for discrepancies of $\left\{\theta^{n} x\right\}$. Acta Math. Hungar. 118 (2008), 155-170. MR2378547(2008m:60049)

[7] K. Fukuyama, The law of the iterated logarithm for the discrepancies of a permutation of $\left\{n_{k} x\right\}$. Acta Math. Hungar. 123 (2009), 121-125. MR2496484 (2010c:11093)

[8] K. Fukuyama and K. Nakata, A metric discrepancy result for the Hardy-Littlewood-Pólya sequences. Monatshefte Math. 160 (2010), 41-49. MR2610311

[9] K. Fukuyama and B. Petit, Le théorème limite central pour les suites de R. C. Baker. Ergodic Theory Dynam. Systems 21 (2001), 479-492. MR.1827114 (2002e:11099)

[10] I.S. Gál, A theorem concerning Diophantine approximations, Nieuw Archief voor Wiskunde 23 (1949), 13-38. MR0027788 (10:355a)

[11] V.F. Gaposhkin, The central limit theorem for some weakly dependent sequences. Theory Prob. Appl. 15 (1970), 649-666.

[12] M. Kac, On the distribution of values of sums of the type $\sum f\left(2^{k} t\right)$. Ann. of Math. 47 (1946), 33-49. MR0015548 (7:436f)

[13] A. Khinchin, Ein Satz über Kettenbrüche mit arithmetischen Anwendungen. Math. Zeitsch. 18 (1923), 289-306. MR 1544632

[14] E. Landau, Vorlesungen über Zahlentheorie, Vol. 2, S. Hirzel, Leipzig, 1927.

[15] R. Marstrand, On Khinchin's conjecture about strong uniform distribution. Proc. London Math. Soc. 21 (1970), 540-556. MR0291091 (45:185)

[16] R. Nair, On strong uniform distribution. Acta Arith. 56 (1990), 183-193. MR.1082999 (92g:11076)

[17] W. Philipp, Das Gesetz vom iterierten Logarithmus für stark mischende stationäre Prozesse. Z. Wahrscheinlichkeitstheorie verw. Gebiete 8 (1967), 204-209. MR0215361 (35:6202)

[18] W. Philipp, Empirical distribution functions and strong approximation theorems for dependent random variables. A problem of Baker in probabilistic number theory. Trans. Amer. Math. Soc. 345 (1994), 707-727. MR1249469 (95a:11067)

[19] P. Révész, The law of the iterated logarithm for multiplicative systems. Indiana Univ. Math. J. $21(1971 / 72), 557-564$. MR0292139(45:1226)

[20] W. Schmidt, Diophantine approximation. Lecture Notes Math. 785, Springer, 1980. MR568710 (81j:10038)

[21] F. Spitzer, Principles of random walk. Van Nostrand, 1964. MR0171290 (30:1521)

[22] G. Shorack and J. Wellner, Empirical Processes with Applications to Statistics. Wiley, New York 1986. MR838963(88e:60002)

[23] S. Takahashi, A gap sequence with gaps bigger than the Hadamards. Tôhoku Math. J. 13 (1961), 105-111. MR0126650 (23:A3946)

[24] S. Takahashi, The law of the iterated logarithm for a gap sequence with infinite gaps. Tôhoku Math. J. 15 (1963), 281-288. MR0157147(28:384)

[25] A. Thue, Bemerkungen über gewisse Näherungsbrüche algebraischer Zahlen. Skrift Vidensk Selsk. Christ. 1908, Nr. 3.

[26] R. Tijdemann, On integers with many small prime factors, Compositio Math. 26 (1973), 319-330. MR0325549 (48:3896) 
[27] H. Weyl, Über die Gleichverteilung von Zahlen mod. Eins, Math. Ann. 77 (1916), 313-352. MR.1511862

[28] A. Zygmund, Trigonometric series. Vol. I, II. Cambridge Library. Cambridge University Press, Cambridge, 2002. MR.1963498 (2004h:01041)

Department of Mathematics A, Graz University of Technology, Steyrergasse 30 , A-8010 Graz, Austria

E-mail address: aistleitner@math.tugraz.at

Institute of Statistics, Graz University of Technology, Münzgrabenstrasse 11, A8010 Graz, Austria

E-mail address: berkes@tugraz.at

Department of Mathematics A, Graz University of Technology, Steyrergasse 30, A-8010 Graz, Austria

E-mail address: tichy@tugraz.at 